\title{
Some observations on the operant behavior of desert pack rats (Neotoma lepida)
}

\author{
LINCOLN D. CLARK \\ University of Utah School of Medicine, Salt Lake City, Utah 84112 \\ and \\ PATRICIA E. GAY \\ Rutgers University, Camden, New Jersey 08102
}

\begin{abstract}
Four desert pack rats (Neotoma lepida) and four Sprague-Dawley rats were maintained in operant chambers continuously for 6 months and exposed to a variety of experimental conditions. Compared to the Sprague-Dawley rats, Neotoma required a lower food intake to maintain its body weight and emitted responses at a more uniform, distributed rate over 24-h periods. The unusual hoarding drive characteristic of Neotoma could be demonstrated in the operant chambers by dispensing glass beads in the pellet dispenser. However, there was no clear evidence that these "nonorganic rewards" were reinforcing.
\end{abstract}

The species Neotoma, also called the pack rat, shows several interesting features which led us to bring them to the laboratory for systematic study and comparison with standard laboratory rats. As their popular name indicates, hoarding behavior is highly developed. They collect and store not only food, but also are attracted to a variety of trinkets and trash items. During pregnancy, the female becomes particularly possessive and may invade the male's lodging to capture his stores of food for her larder. They also seem to be highly perceptive of minor changes in their environment. In their desert habitat, they build multichambered mound houses. If one removes with forceps a few sticks from the house surface and places them 5-10 ft from the dwelling, the rats will retrieve them and replace them on their house with compulsive accuracy. On the other hand, if one adds additional sticks to the house, they will remove them.

It was felt that the pack rat's phenotypic peculiarities, particularly its unusual acquisitive drives, might be reflected in its operant behavior and pattern of adaptation to the artificial environment of the Skinner box. The following series of exploratory studies were therefore performed. For comparative purposes, Sprague-Dawley rats were also subjected to the same experimental procedures.

This research was supported by NIMH Grant 05739 to Lincoln D. Clark, M.D. We are indebted to Edward Nakashima for technical assistance throughout the course of the research. Requests for reprints should be sent to Lincoln D. Clark, Department of Psychiatry, University of Utah School of Medicine,

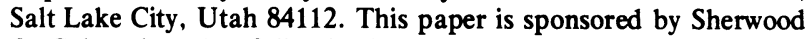
O. Cole, who takes full editorial responsibility.

\section{METHOD AND PROCEDURE}

Four Neotoma lepida, two males and two females, were obtained as young adults and kept in the laboratory for 2 years. These animals had been laboratory raised and were several generations removed from the original wild-trapped stock. When not in test chambers, they were housed in pairs in large (18 $\times 18$ in.) cages and maintained on ad-lib food (Purina Rat Chow) and water. Four Sprague-Dawley rats of the same age and sex were maintained in an identical manner.

During the studies being reported, the animals were housed in operant chambers continuously for 6 months. The chambers were similar to the standard Grason-Stadler rodent chambers. They were equipped with two levers located 3 in. above floor level. Noyes pellets $(45 \mathrm{mg}$ ) and other items could be dispensed automatically into a recessed cup between the two bars at floor level. In addition, a small cup for "collecting and storing" items was provided near the foodcup. Water was accessible at all times via a drinking tube attached to one side of the chamber and cotton batton was provided for bedding. Chambers were on a 12-h light, 12-h dark cycle. Responses and reinforcements were recorded continuously for each lever on Harvard cumulative recorders and printed out cumulatively every $2 \mathrm{~h}$ on Pressin printout counters. Animals were weighed once a week, the only time when they were removed from the chambers.

At the beginning of the study, the animals were removed from their home cages, where they had had free access to pelleted food, and placed in chambers scheduled to reward $100 \%$ of presses on either bar (CRF schedule of reinforcement). No shaping was done. The purpose was to determine if there were differences in "spontaneous" rates of acquiring the barpressing response. Animals were maintained on this schedule for 4 weeks.

At the end of the 4 weeks, an ad-lib supply of standard rat pellets was placed in each chamber and the animals were provided with a choice of "free" or "worked for" food. This test lasted for 8 days.

On the next day, the ad-lib supply of food was removed and Neotoma and Sprague-Dawley were compared on a series of fixed-ratio (FR) schedules with $100 \%, 50 \%, 25 \%$, and $5 \%$ of responses rewarded, and later on a fixed-interval (FI) schedule with reinforcements available every $60 \mathrm{sec}$. Subsequently, a series of reversal experiments were carried out. These involved alternately inactivating one lever and providing continuous reinforcement (CRF) on the other. One series of reversals was carried out with 1 - 
week irtervals between reversals; in another, levers were reversed every other day. Sixteen reversals were made in each set. It was hoped that this procedure would provide information on rate of acquisition of learning sets as well as frequency of perseverative errors with infrequent as opposed to more frequent reversals.

In the last phase of the study, various proportions of colored glass beads were mixed with the $45-\mathrm{mg}$ pellets in the hopper of the dispenser to be delivered randomly in response to barpressing. During this phase, both bars were active and programmed for continuous reinforcement.

\section{RESULTS AND DISCUSSION}

When animals were introduced to the test situation, without deprivation, the Neotoma invariably discovered the reinforcement mechanism within the first $24 \mathrm{~h}$ and were working at $80 \%$ of their eventual mean daily response rate within 3 days. SpragueDawley made a few scattered responses during the first $48 \mathrm{~h}$, but a clear upward trend in response rate did not appear until the third day. This difference is difficult to interpret. One posiible explanation is that Neotoma, before driven by severe hunger, responds to a novel environment with more inquisitive, exploratory behavior and, therefore, "discovers" the reward mechanism sooner.

Both species were able to maintain themselves in good nutritional state (stable body weight) on both the FR and FI schedules used. However, in terms of pellet consumption per kilogram of body weight, Neotoma consumed $24 \%$ less food than Sprague-Dawley rats. In that the two species were on identical work schedules and did not appear strikingly different in general activity, the former would appear to be metabolically more efficient.

The pattern of barpressing for food was also of interest. Both species showed considerable variability from day to day in terms of the number of pellets taken per $24-h$ period. However, the daily variation from the group means calculated for the entire experiment was within narrower limits for Neotoma. The tendency for this species to work at a more constant but slower rate appeared in the distribution of responses within successive 24 -h periods. Neotoma worked relatively steadily and responses were distributed fairly uniformly over successive 2-h intervals recorded. Sprague-Dawley worked in bursts, with peaks of high performance followed by relative inactivity, a pattern of feeding behavior previously described for the laboratory rat (e.g., Baker, 1953; Balagura \& Coscina, 1968). Both species showed higher levels of performance nocturnally, but diurnal patterns were most striking in Sprague-Dawley. Neotoma made a higher percentage of responses during daylight. When both levers were activated, Neotoma showed a more striking side preference, generally right-handed, than Sprague-Dawley, which distributed responses more equally between levers.

When animals were allowed to choose between "free" and "worked for" food, Sprague-Dawleys chose the former and only sporadic barpresses occurred. In the Neotoma group, response rates dropped to near zero for $8-10 \mathrm{~h}$ after introduction of the pellets. Neotoma then returned to a fairly steady rate of barpressing for food which averaged (over 8 days) $30 \%$ of their response rate when on pellet dispenser feeding alone.

The species were similar in acquiring the scalloped response pattern characteristic of an FI schedule. The differences which appeared were in terms of consistency of performance. After equivalent training, more typical deep scallops were present in the cumulative records of Neotoma performances. This was reflected in a consistently higher percentage of responses printed out for the second half as compared with the first half of the $60-\mathrm{sec}$ FI interval.

Both species, on successive lever reversals, showed acquisition of a learning set. When the percentage of correct responses for the $6 \mathrm{~h}$ after reversal was plotted, the efficiency of making a reversal became asymtotic at less than $10 \%$ errors by the eighth reversal. The results of the experiment in which reversals were introduced every $48 \mathrm{~h}$ is presented in Figure 1. The curves plotted as group means were not appreciably different. However, when the range of percent correct responses was plotted (as in Figure 1), the greater uniformity of performance of Neotoma became evident.

Neither species hoarded food pellets obtained through barpressing either in their nest or in the storage cup provided in the chamber. When beads were mixed with the pellets and delivered into the food tray, Neotoma retrieved and stored considerable numbers of them in the cup. No systematic bead

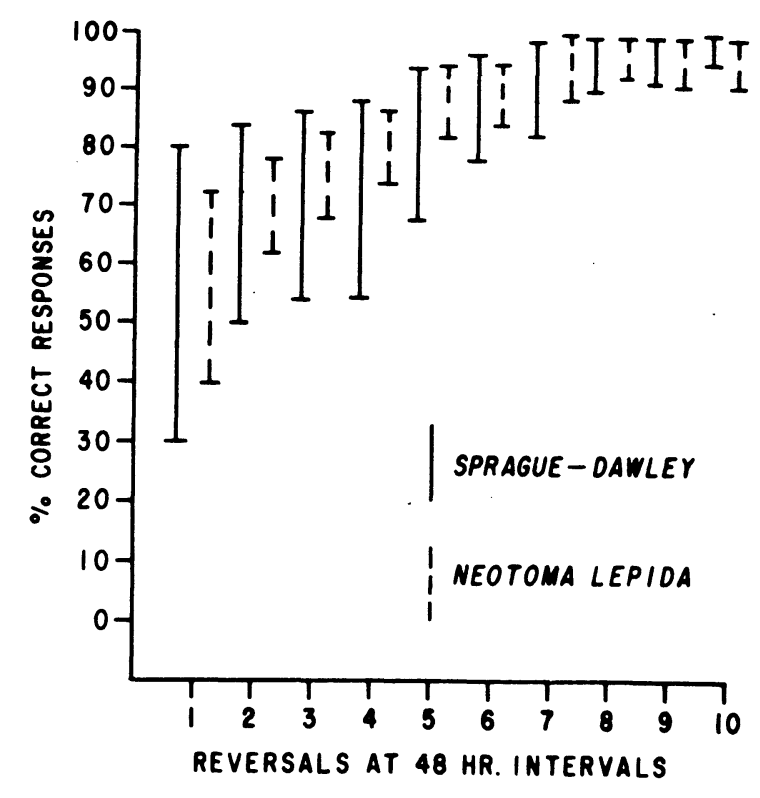

Figure 1. Learning set acquisition in Neotoma lepida and Sprague-Dawley rats. Bars indicate the range of performance for the four animals in each group. 
hoarding was seen in Sprague-Dawley control rats. Both species adjusted their response rates upward to compensate for the percentage of beads mixed with the pellets. With one exception, there was no evidence that these objects per se reinforced barpressing (i.e., rates of response did not increase beyond that required to maintain a constant food intake) under "normal" conditions. In female Neotoma, however, phasic increases in hoarding related to estrous cycles were observed. Response rates during these times were clearly higher than at comparable periods when beads were not dispensed. When other objects were not available, Neotoma used the cup to store dry fecal boli retrieved from the nest. For obscure reasons, both beads and fecal boli were stored only temporarily. Periodically, the contents of the cup would be found emptied into the refuse tray and another period of accumulation would begin.

From the beginning, it was recognized that these studies were exploratory in nature and had serious limitations from the standpoint of comparative analysis of behavior. If one compares in experimental tests two different species of rodents, especially when one is close to its wild origins and the other long domesticated for laboratory use, the discovery of behavioral differences is not surprising, although the reasons for the differences may be difficult to explain. Knowledge of the phenotypically characteristic behavior of the animal in its natural habitat, however, may provide important clues. For example, the rate of acquiring pole escape responses and their resistance to extinction are greater in two subspecies of deer mice, gracilis and sonoriensis, which are semi- arboreal in habitat, than in earth-bound subspecies of deer mice (Wolfe, Swinyard, \& Clark, 1962).

It was felt that the pack rat's phenotypic peculiarities, particularly its unusual acquisitive drives, might be reflected in its operant behavior and adaptation to the artificial environment of the operant chamber. These as well as other interesting differences were indeed found. In comparison with Sprague-Dawley rats, Neotoma (1) more rapidly discovered the technique for acquiring food rewards in the artificial environment, (2) required less food to maintain body weight, (3) showed less diurnal variation in eating activity, (4) showed more uniformity in work output, and (5) continued operant work for food even in the presence of "free" food. Neither species hoarded food pellets obtained through operant work. Neotoma did, however, store and periodically discard "nonorganic" rewards in the form of glass beads admixed with dispensed food pellets.

\section{REFERENCES}

Baker, R. A. Aperiodic feeding behavior in the albino rat. Journal of Comparative and Physiological Psychology, 1953. 46. $422-426$.

Balagura, S., \& Coscina, D. V. Periodicity of food intake in the rat as measured by an operant response. Physiology and Behavior, 1968, 3, 641-643.

Wolfe, H. H., Swinyard, E. A., \& Clark, L. D. The differential effects of chlorpromazine and pentobarbital on two different forms of conditioned avoidance behavior in Peromyscus maniculatus gracilis. Psychopharmacologia (Berlin), 1962, 3, 438-444.

(Received for publication June 14, 1976.) 